\title{
Tracheal ultrasound to assess endotracheal tube depth: an exploratory study
}

\author{
William P. McKay, MD • Andrew Wang, MD • \\ Kinsha Yip, MD · Mateen Raazi, MBChB
}

Received: 9 December 2014 / Accepted: 12 January 2015/Published online: 22 January 2015

(C) Canadian Anesthesiologists' Society 2015

\section{To the Editor,}

This letter outlines an observational study to assess endotracheal tube (ETT) depth using ultrasound. Ideal ETT tip position is in the mid-trachea, and malpositioning can result in unintended extubation, hypoxia, or barotrauma. ${ }^{1}$ Transverse plane ultrasound of the saline-filled ETT cuff has been reported to determine ETT depth in children and esophageal placement in adults. ${ }^{2,3}$ Although the use of measurement marks (MM) at the teeth $(21 \mathrm{~cm}$ for females, $23 \mathrm{~cm}$ for males) is simple and commonly employed, this method is unreliable for ensuring adequate ETT tip placement. $^{4}$

We explored the concept of ETT positioning using longitudinal ultrasound while watching the movement (undulation) of the tracheal rings as the ETT tip slides forward. We hypothesized that ultrasound observation of the ETT tip while it is moving slowly down the trachea would improve correct placement (ETT tip $>2.5 \mathrm{~cm}$ from the carina with the cuff still below the cords) compared with the conventional MM technique.

With University of Saskatchewan Ethics Board approval (May 2012), consent was obtained from adult elective surgery patients where routine tracheal intubation was planned. Non-elective and physiologically unstable patients were excluded. Following induction of anesthesia, the attending anesthesiologist was instructed to "advance the ETT slowly once the tip is through the

\footnotetext{
W. P. McKay, MD (ه) · A. Wang, MD · K. Yip, MD .

M. Raazi, MBChB

Department of Anesthesiology, Perioperative Medicine and Pain

Management, University of Saskatchewan, Saskatoon, SK,

Canada

e-mail: bill.mckay@usask.ca
}

cords" while an investigator viewed the trachea in the long axis using ultrasound (MicroMaxx ${ }^{\circledR}$ with MSK L25e/13-6 $\mathrm{MHz}$ transducer; SonoSite Inc. Bothell, WA, USA). Disposable cuffed ETTs (8-mm internal diameter for females, 9-mm for males) were used for all intubations. When the ultrasound image of the tracheal ring undulation - caused by the advancing ETT - showed that the ETT tip had reached the sternal notch, the ETT was fixed at that depth. In addition, the depth marking at the upper incisors was noted in order to calculate where the ETT tip placement would have been if MM had been used. The ETT was carefully kept at that depth while an investigator evaluated (using flexible bronchoscopy) the ETT depth as "too shallow", "too deep", or "correct". A Chi square test was used to compare the MM vs the ultrasound method and intent-to-treat analysis was performed on the bronchoscopic evaluations. A sample size of 40 patients was based on a previous study. ${ }^{5}$

Fifty-three patients were approached; three declined consent, and seven were excluded because a bronchoscope was not available. This left 43 subjects ( 24 females and 19 males) to be evaluated. Sixteen patients had a BMI > $30 \mathrm{~kg} \cdot \mathrm{m}^{-2}$, including four subjects with a BMI > $40 \mathrm{~kg} \cdot \mathrm{m}^{-2}$. Movement of the ETT tip was visualized in $42(98 \%)$ subjects. Using ultrasound resulted in 38 of 43 $(88 \%)$ patients with a correct ETT position compared with $24(56 \%)$ correct if only the conventional MM method had been used (mean difference $32 \%$; $95 \%$ confidence interval [CI] 18 to $46 \% ; P=0.002)$. There were no endobronchial intubations, whereas two would have resulted with MM.

Tracheal ultrasound was more successful than MM for proper ETT tip placement, but tracheal ultrasound was similar to results using palpation of the ETT tip during advancement, as previously reported, which resulted in $85 \%$ (95\% CI 77 to 92 ) correct tip placements. ${ }^{5}$ 
Though this study had the pragmatic strength of being conducted in a real-world clinical situation, none of the investigators were experienced sonographers, which may have affected the efficacy of the ultrasound. The study had some limitations; for example, the MM was calculated and not used as an a priori guide for ETT depth. Nevertheless, the validity of the calculation is plausible, since changing the ETT depth at the teeth would be expected to result in the same change at the tip.

These data suggest that tracheal ultrasound of the advancing ETT tip is preferable to the conventional MM method for assessing ETT depth. It is uncertain whether tracheal ultrasound is preferable to tracheal palpation alone, which requires no ultrasound equipment.

Acknowledgment This study was funded by the Department of Anesthesiology, University of Saskatchewan. No author has any commercial or other affiliations that are, or may be perceived to be, a conflict of interest.

\section{References}

1. Rudraraju P, Eisen LA. Confirmation of endotracheal tube position: a narrative review. J Intensive Care Med 2009; 24: 283-92.

2. Das SK, Choupoo NS, Haldar R, Lahkar A. Transtracheal ultrasound for verification of endotracheal tube placement: a systematic review and meta-analysis. Can J Anesth 2015; DOI:10. 1007/s12630-014-0301-z.

3. Tessaro MO, Salant EP, Arroyo AC, Haines LE, Dickman E. Tracheal rapid ultrasound saline test (T.R.U.S.T.) for confirming correct endotracheal tube depth in children. Resuscitation 2014; DOI:10.1016/j.resuscitation.2014.08.033.

4. Sitzwohl C, Langheinrich A, Schober A, et al. Endobronchial intubation detected by insertion depth of endotracheal tube, bilateral auscultation, or observation of chest movements: randomised trial. BMJ 2010; 341: c5943.

5. McKay WP, Klonarakis J, Pelivanov V, O'Brien JM, Plewes $C$. Tracheal palpation to assess endotracheal tube depth: an exploratory study. Can J Anesth 2014; 61: 229-34. 